\title{
Molecular Opacities in Cool Dwarf Stars
}

\author{
James Liebert \\ Steward Observatory, University of Arizona, Tucson AZ 85721, USA
}

\section{Introduction}

The term dwarf stars identifies objects of small radius in the Hertzsprung-Russell (H-R) Diagram, but encompasses more than one phase of stellar evolution. The $M$ dwarfs (type $\mathrm{dM}$ ) populate the main sequence at the low mass end; these are the coolest core hydrogen-burning stars. They belong generally to the Galactic disk, or Population I, have relatively small space motions with respect to the Sun, and have similar metallicities to the Sun (although perhaps only within a factor of several). In particular, this means that the abundance of oxygen is always greater than that of carbon. The M subdwarfs (sdM) are the Population II counterparts, showing low metallicities and high space motions. Because they have smaller radii, they define a main sequence at lower luminosity than the $M$ dwarfs for a given temperature. Hence the term subdwarf.

A third type of dwarf star is the white dwarf. This is an object which has ended its nuclear-burning life, and has contracted into an electron-degenerate configuration the size of the Earth, most likely with a core composed of the products of helium-burning - carbon and oxygen. Sometimes called degenerate dwarfs, these objects fall along a diagonal cooling track in an H-R Diagram. They range in temperature (luminosity) from higher than $100,000 \mathrm{~K}$ (above solar luminosity) to perhaps $4,000 \mathrm{~K}\left(<10^{-4} L_{\odot}\right)$, depending on how long the object has been cooling at a constant radius.

Likewise, there may be objects called brown dwarfs, too low in mass to initiate hydrogen burning. They radiate first by the release of half the energy of gravitational contraction, may initiate limited cycles of nuclear-burning for a finite period, after which they enter a cooling track for hydrogen-rich configurations; they have roughly ten times the radii of carbon-oxygen degenerate dwarfs.

Finally, in the last two decades, a fifth category has been recognized - the carbon dwarf. This is a low mass main sequence star whose carbon abundance exceeds the oxygen abundance. Normally, in the $\mathrm{dM}$-sdM atmospheres the carbon is locked up in $\mathrm{CO}$ and a few other molecules. In the dwarf carbon $(\mathrm{dC})$ stars, it is the oxygen that is locked up in $\mathrm{CO}$ while the spectra reveal a rich chemistry of carbon-based molecules. 
In the following sections, I will review the properties of the spectra and the analyses for each of these categories except for the white dwarfs (see Thejll this volume).

\section{M Dwarf and Subdwarf Stars}

There has been relatively little attention given to studying the spectra and atmospheres of the dM stars, less so even than for $\mathbf{M}$ giants. With relatively low temperatures and high pressures, these atmospheres are fertile ground for molecule formation. Given the modest amount of quantitative data for molecules more complex than diatomic, one might fear that our ability to match observations with synthetic spectra may be quite limited for this type of star. Indeed, up until recently, very few relevant model atmosphere calculations even existed, and researchers routinely estimated the temperatures of these stars by fitting colors (and/or spectra) to blackbodies. As we shall see, the energy distributions of these stars are very unlike that of a blackbody.

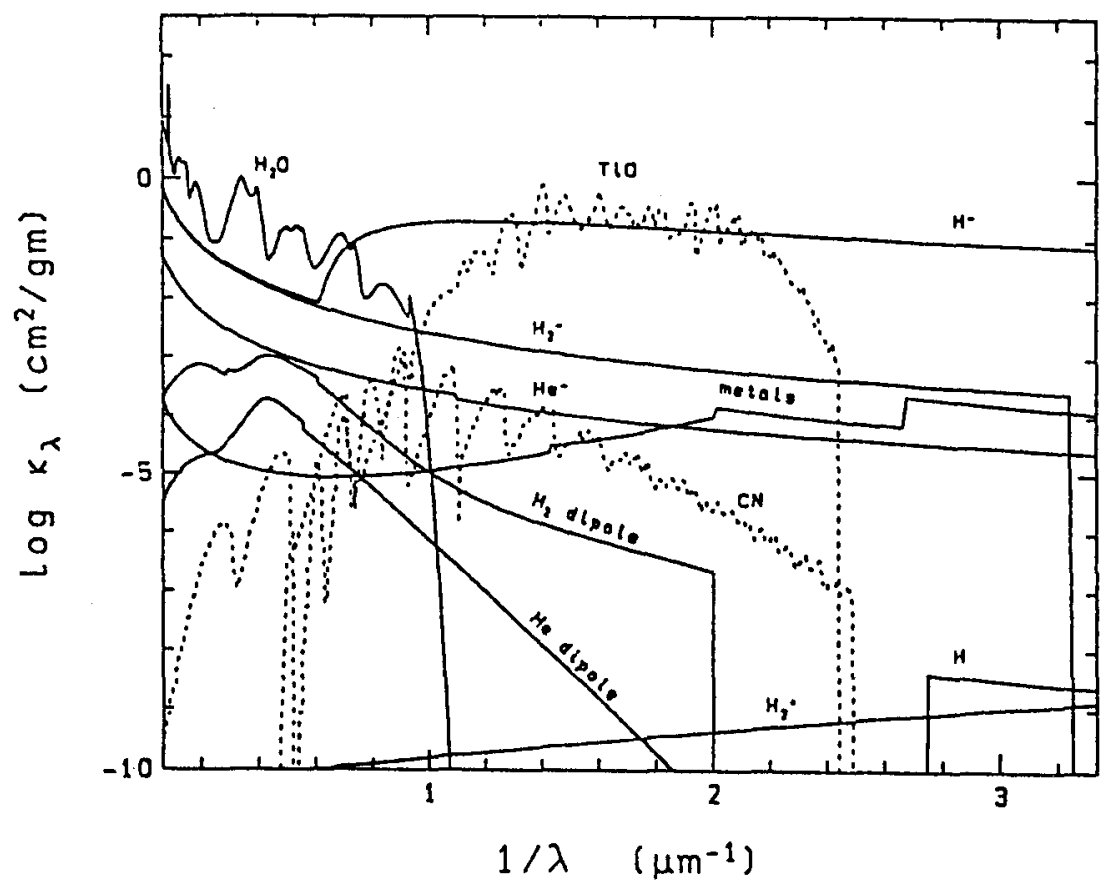

Fig. 1. Opacities calculated for various molecular and atomic constituents plotted against wave number for a $3000 \mathrm{~K}, \log \mathrm{g}=5$, solar composition model from Allard (1990). Note the prominent role of $\mathrm{H}_{2} \mathrm{O}, \mathrm{TiO}$ and $\mathrm{H}^{-}$. 
Fortunately, in the last few years the situation has changed dramatically for the better. This is primarily due to two PhD dissertations - by Allard (1990) at the University of Heidelberg, and by Ruan (1991) at Mt. Stromlo Observatory and the Australian National University. Allard (1994) adds the exclamation point to some of the following comments concerning her dissertation and some subsequent work. Unfortunately, neither Allard's nor Ruan's dissertation have been published.

The molecular (and atomic) opacities important in $\mathbf{M}$ dwarf stars are well illustrated in Fig. 1, taken from Allard's (1990) thesis, for a model at 3,000 K, log $\mathrm{g}=5$, and solar composition. Two of the dominant opacity sources are the $\mathrm{TiO}$ molecule in the optical and $\mathrm{H}_{2} \mathrm{O}$ in the infrared. Dominant continuous opacity sources include $\mathrm{H}_{2}^{-}$in the infrared and $\mathrm{H}^{-}$in the optical.

For low metallicity stars (the subdwarf $\mathrm{M}$ ), the pressure-induced dipole opacities due (see Borysow this volume) due to $\mathrm{H}_{2}-\mathrm{H}_{2}$ and $\mathrm{H}_{2}-\mathrm{He}$ can dominate the infrared opacity, while $\mathrm{H}^{-}$is strongest in the optical. Molecules involving heavier elements are almost completely restricted to hydrides - $\mathrm{MgH}, \mathrm{CaH}$ being the strongest in the optical spectrum.

What can we say about the accuracy of these models and the fits of synthetic to observed spectra? Certainly, a number of improvements to the physics have been made in recent years. It appears that these models now can yield improved estimates of the effective temperature $\left(T_{\text {eff }}\right)$ of low mass stars.

Kirkpatrick et al. (1993a) made the first comprehensive attempt to fit a sequence of $M$ dwarf spectroscopic standards, from early $M$ spectral types at $\geq 3500 \mathrm{~K}$ to as low as $2700 \mathrm{~K}$. Two examples of these fits are shown as Fig. 2. The overall energy distribution is fit rather well, though the quantitative fits to $\mathrm{TiO}$ and other band strengths are only fair, and become worse at shorter wavelengths.

Since all of these near neighbors of the Sun have excellent trigonometric parallax measurements, the luminosities of the stars can be determined rather
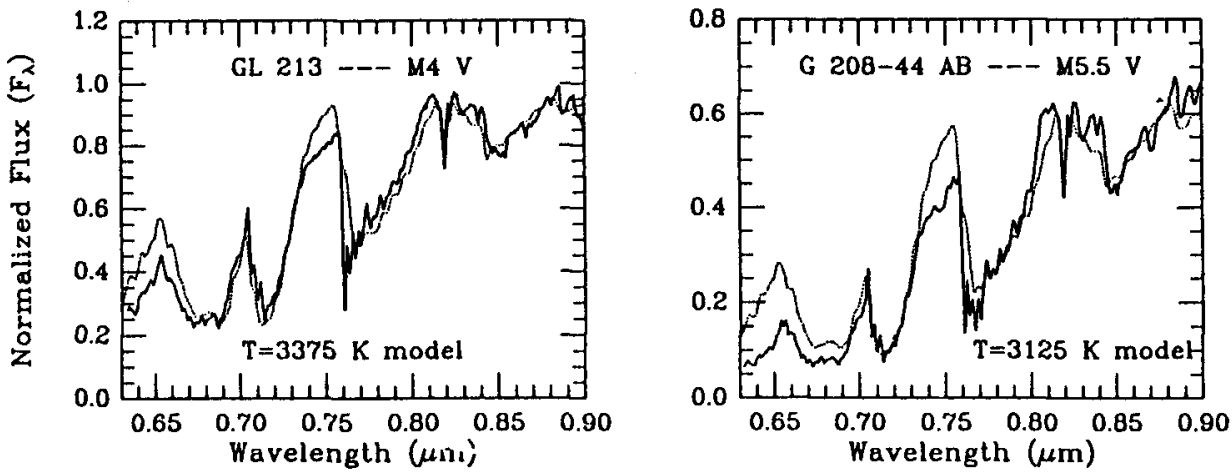

Fig. 2. Two examples of fits to optical spectra of middle $M$ dwarfs using Allard models, from Kirkpatrick et al. (1993a). 
accurately once the temperatures are estimated. The derived positions in a Hertzsprung-Russell ( $\log L$ vs. $\log T_{\text {eff }}$ ) diagram can then be compared to the predictions of stellar interiors models for main sequence stars of very low mass.

When this comparison was made using $T_{\text {eff }}$ values estimated by fitting blackbodies, the observed stars appeared usually to have smaller $T_{\text {eff }}$ at a given luminosity - or alternatively a larger radius - than predicted by the interiors models (see Burrows \& Liebert 1993, Fig. 19). This implied either that calculated atmospheric fits were wrong, the interiors models were wrong, or that many low luminosity stellar objects in the solar neighborhood were in fact substellar mass "brown dwarfs."

Using the Allard atmosphere models, however, Kirkpatrick et al. (1993a) find essentially a $T_{\text {eff }}$ scale for the sequence of $\mathbf{M}$ dwarfs which is in fair agreement with the interior models - see Fig. 3. This implies that the atmospheric and interiors models are consistent with each other and with the assumption that the observed objects are hydrogen-burning stars ( $M$ dwarfs). However, the agreement was still poor for the lowest mass stars with $T_{\text {eff }} \leq 3,000 \mathrm{~K}$.

Improvements made since this 1994 paper (see Allard 1994) include notably the addition of FeH bands near one micron in the spectrum and the treatment of

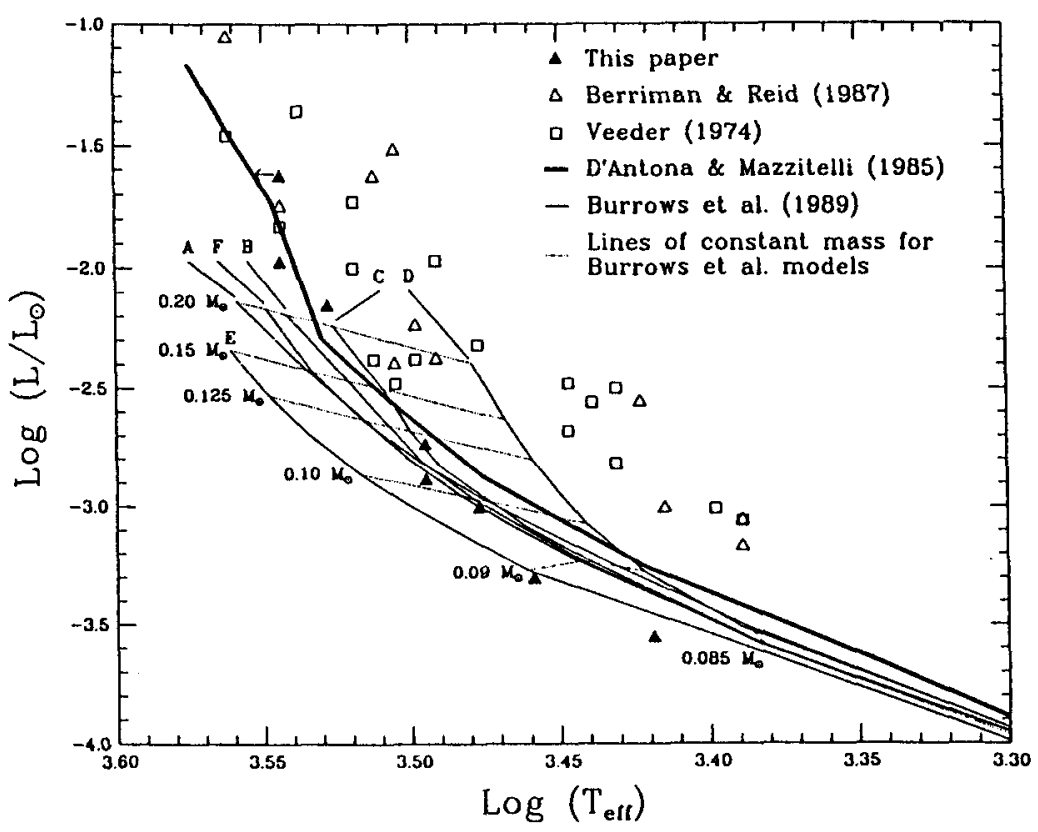

Fig. 3. Temperatures and luminosities for the $M$ star standard sequence of Kirkpatrick et al. (1993a) (filled circles) are compared with previous determinations (open symbols) and various stellar interiors models (curves) for main sequence stars, with mass points labelled. 
the pressure-induced dipole opacities by Lenzuni et al. (1991). Now the synthetic spectrum fit to one of the lowest luminosity stars in the Kirkpatrick et al. (1993a) is much improved for $T_{\text {eff }}$ of $2,800 \mathrm{~K}$; the fit has now been extended to include observations to $3.5 \mu$ by Jones et al. (1993) (see also review by Miller et al. in this volume). The complicated band spectrum of $\mathrm{H}_{2} \mathrm{O}$ remains the important piece which does not fit well.

Can we now be confident of the atmospheric and stellar paramaters derivable for low mass stellar spectra? Such an attitude would be premature. The models predict far too much flux in the visual and bluer wavelengths, which must tell us that there are still many opacity sources to be added to the models. A similar problem persists with attempts to fit subdwarf $M$ stars with models having drastically reduced metallicities. The missing components undoubtedly are primarily molecular in nature, and for the subdwarfs AlH may be particularly important at blue wavelengths. But we are off to a good start, finally, with M dwarf stars.

\section{Brown Dwarf Candidates}

Not a single object has yet been discovered outside of our solar system (but not including evolved, binary systems) that can unambiguously be proclaimed a substellar object. However, this situation is arguably due to the fact that it is so difficult to prove that a given candidate is a brown dwarf, and does not necessarily mean that substellar objects do not exist. Indeed, numerous candidates exist, and more are being found at a rapid rate. A detailed review of the candidates and the methods of discovery is given in Burrows \& Liebert (1993).

Here I wish to discuss only two of the candidates - among the few for which more than a little spectrophotometric information exists. The first, GD165B, is a spatially-resolved companion to a white dwarf star and clearly has a much lower temperature than any visible object yet known (Becklin \& Zuckerman 1988). A poor-quality optical spectrum (Kirkpatrick et al. 1993b) appears to differ qualitatively from those of the least luminous known stars - see Fig. 4 taken from that paper, Davis (this volume) discuss the possibility that a band system of VO, expected to grow stronger at cooler $T_{\text {eff }}$, may explain the enhanced absorption near $8500 \AA$ found by Kirkpatrick et al. (1993b).

The observations are not yet accurate enough for the $T_{\text {eff }}$ of GD165B to be estimated, although the infrared colors suggest it is at least several hundreds of degrees cooler than vB10, for example. Still, the implied luminosity is nearly $10^{-4}$ $L_{\odot}$, and is consistent with high opacity main sequence models near the stellar mass limit of $0.075-0.08 M_{\odot}$. GD165B could be a hydrogen-burning star, but then very likely it would be the lowest mass star known. A second brown dwarf candidate for which considerable (and very confusing!) information exists is G29$38 \mathrm{~B}$, another potential companion to a white dwarf discovered by the same group (Zuckerman \& Becklin 1987). I say "potential companion" because what these authors found was that the white dwarf has a spatially-unresolved infrared 


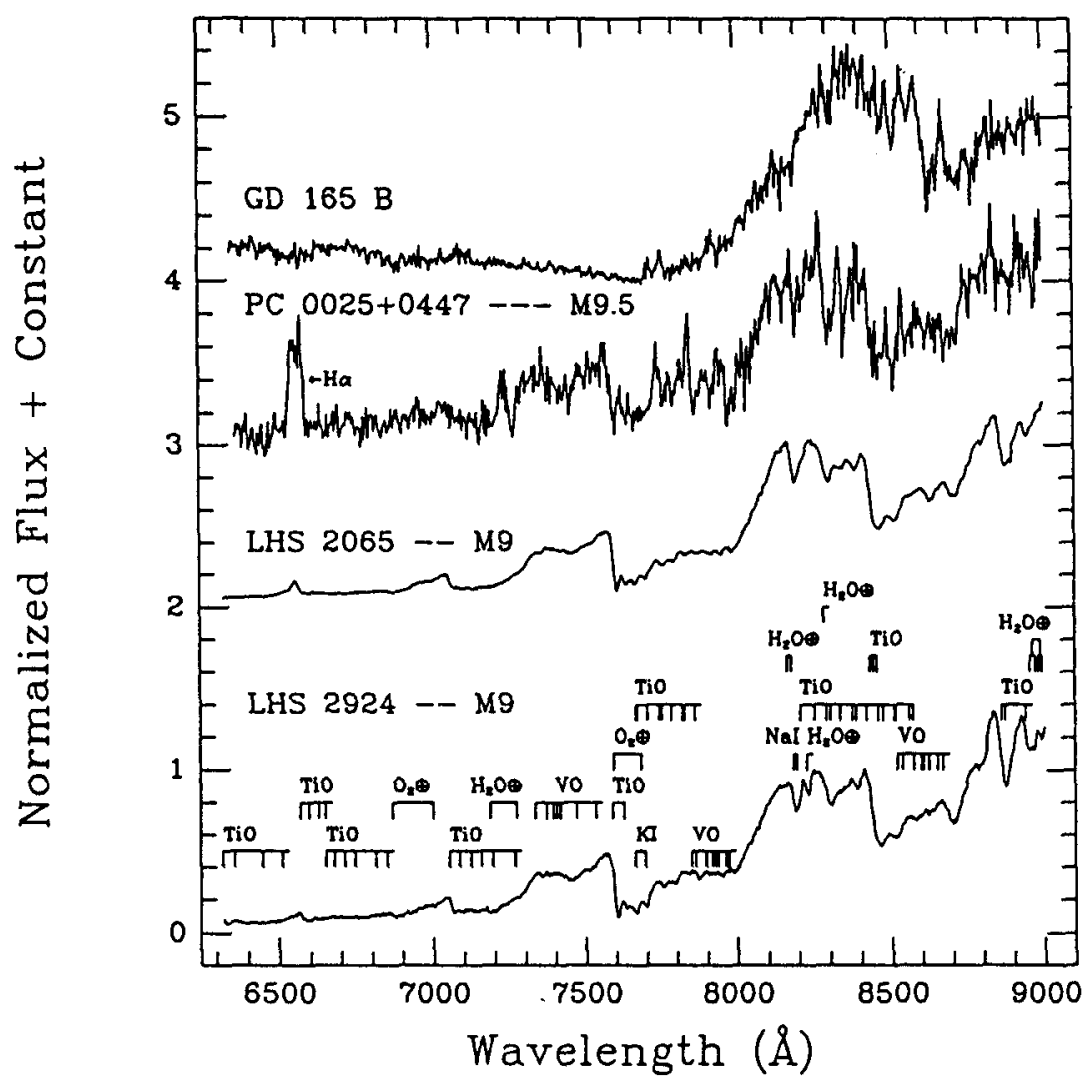

Fig. 4. Spectra of four of the coolest M dwarf stars known, the top of which, GD165B, is discussed in the text. Molecular band features are labelled for LHS2924 (from Kirkpatrick et al. 1993b).

excess, which they initially suggested might be a brown dwarf companion having a $T_{\text {eff }}$ of about $1,200 \mathrm{~K}$ and a considerable luminosity of $5 \times 10^{-5} L_{\odot}$, implying a radius not too different from that expected for a brown dwarf. (Brown dwarfs from $0.001 M_{\odot}$ to stars of $0.08 M_{\odot}$ basically should have a radius similar to that of Jupiter similar to that of Jupiter which is $0.1 R_{\odot}$ ). These and subsequent authors have detected the excess out to wavelengths as long as ten microns, and the flux at these long wavelengths is far in excess of that expected from the above $T_{\text {eff }}$. Further attempts to spatially resolve the infrared source from the white dwarf have failed, and infrared spectra show no evidence for spectral features. Hence, it has been proposed that the excess is due to some kind of dust shell around the white dwarf. However, it should be noted that the white dwarf has a cooling age of order $10^{9}$ years, and may show small amplitude radial velocity variations consistent with having a companion of substellar mass. This complicated situation is reviewed more extensively by Zuckerman (1992), from 
which more detailed references to the work on G29-38 may be found.

Numerous brown dwarf candidates are apparently being found as members of young clusters, including some imbedded in giant molecular clouds and other regions of recent star-formation - see Zinnecker et al. (1993) and Burrows \& Liebert (1993).

I wish to promote the bias that the cup is best considered half full in the widening search for brown dwarfs. To be sure, the optimist must acknowledge that there have been several false claims, missteps, and some null results. But the biggest problem is how we can recognize a brown dwarf, if we find it, from an energy distribution or spectrum. This may require the calculation of spectra and energy distributions for objects with $T_{\text {eff }}$ of 1,000-2,000 K. Such atmospheres would have some properties intermediate between those of Jovian planets and low mass stars, as explored by Lunine et al. (1986) and by Tsuji (this volume). Needless to say, different molecular opacities will be important in trying to calculate an emergent spectrum in this intermediate temperature range. Substellar atmospheres may be a challenging new subject in the decades to come.

\section{Dwarf Carbon Stars}

The first carbon dwarf, listed in the Lowell Observatory proper motion catalogue as G77-61, was discovered by Dahn et al. (1977) amongst the few hundred nearby stars with trigonometric parallax measurements in the U.S. Naval Observatory program. For more than ten years, this 13 th magnitude star remained unique among low mass dwarf stars in showing strong $\mathrm{C}_{2}$ and $\mathrm{CH}$ with weak $\mathrm{CN}$, instead of the normal $\mathrm{TiO}$ features. This implied that most oxygen was tied up in $\mathrm{CO}$ molecules, instead of most of the carbon. At $M_{b o l} \sim+9$, it has the luminosity of an early $\mathbf{M}$ or late $\mathrm{K}$ dwarf, a bit more than $10^{-2} L_{\odot}$, and $T_{\text {eff near }} 4,000 \mathrm{~K}$.

Its existence posed a problem for stellar evolution: While G77-61 has halolike space motions and apparently a very low abundance of heavy elements (Gass et al. 1988), Population II stars consistently show higher $\mathrm{O} / \mathrm{C}$ ratios than stars of higher metallicity. Thus it is very unlikely that such a star could form out of material with $\mathrm{C} / \mathrm{O}>1$. The normal explanation for luminous carbon stars is that these are asymptotic giant branch (AGB) objects with carbon-oxygen cores powered by both hydrogen- and helium-burning shell sources (see Lambert this volume). Due to mixing processes associated with thermal flashes of the heliumburning shell, some carbon from the stellar core gets dredged up into the outer envelope and stellar atmosphere, inverting the $\mathrm{O} / \mathrm{C}$ ratio.

However, it has been known for a long time that there are carbon stars too low in luminosity to be in such an advanced phase of evolution. The subgiant $\mathrm{CH}$ stars, and related types of stars, have generally turned out to be binary; this permits the explanation that a now-unseen companion transferred material to the now-visible star when in the carbon AGB phase. And, sure enough, G77-61 turned out to be a 243-day spectroscopic binary; its likely white dwarf companion is too cool for detection, even with the International Ultraviolet Explorer (IUE) Observatory (Dearborn et al. 1986). 

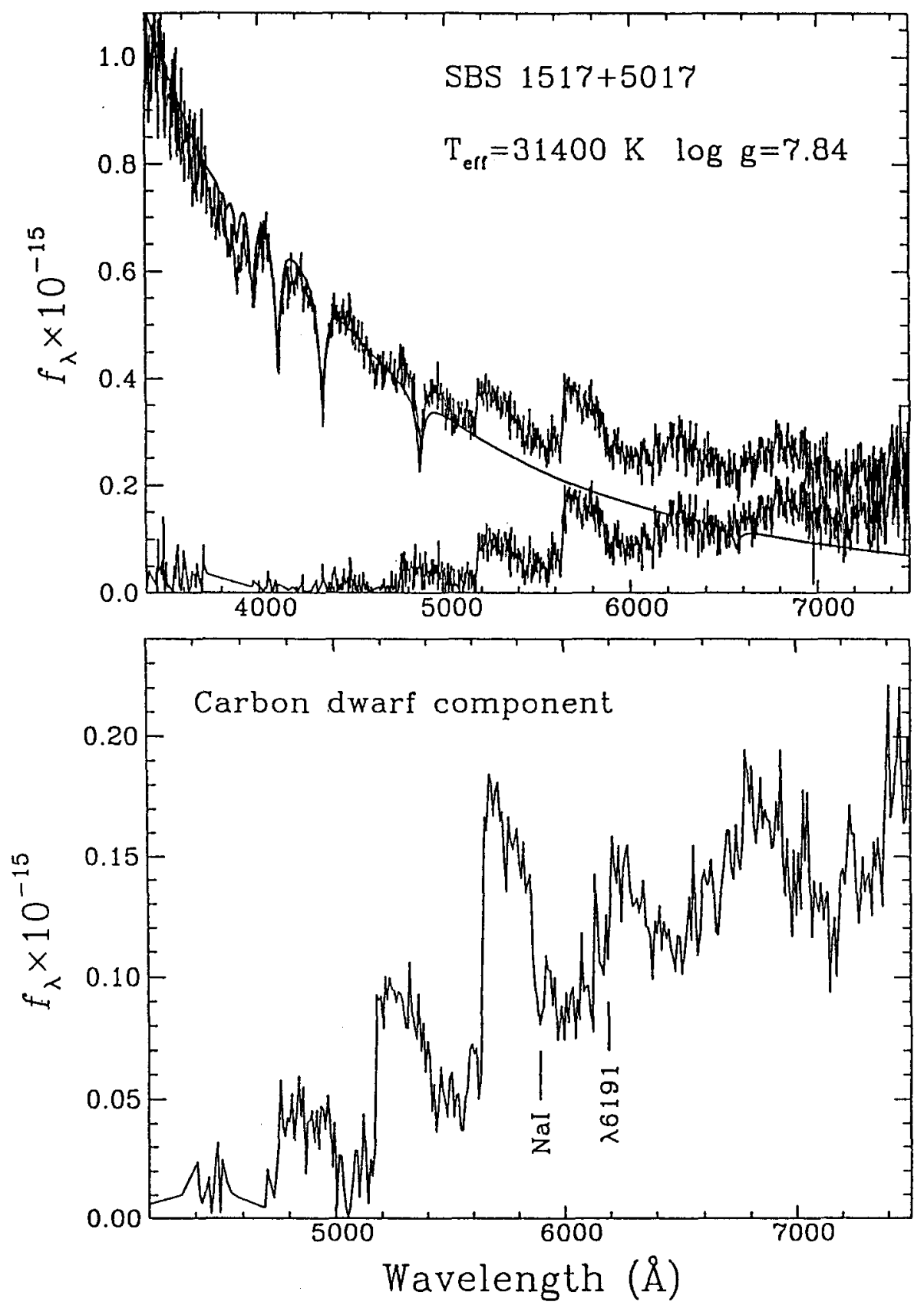

Fig. 5. (a) (top) composite spectrum of DA + dC components; (middle curve) best fitting white dwarf atmosphere model; (bottom) resultant spectrum of carbon dwarf star, as described in text; (b) carbon dwarf spectrum on an expanded scale. 
In the last few years, new discoveries have swelled the number of dwarf carbon stars to ten (cf. Green, Margon and MacConnell 1992; Warren et al. 1993). They all appear to be remarkably similar in $T_{\text {eff }}$ and luminosity to G77-61. Most, though apparently not all of these, show the kinematics of halo or at least old disk stars, though none of the new discoveries have abundance analyses. It is not known whether all are binaries.

In Fig. 5, I illustrate the spectra of the dwarf carbon stars with the latest discovery - called SBS 1517+5017 from the Russian Second Byurakan Survey (Markarian \& Stepanian 1983). This is one of two known cases where a white dwarf companion is actually visible (Liebert et al. 1994). The blue side of this composite optical spectrum (top tracing of the figure) is dominated by the broad hydrogen lines of the DA white dwarf, the hot component of the system. This component can be modelled accurately with an atmosphere having $T_{\text {eff }}=$ $31,300 \mathrm{~K}$ and $\log g=7.84$ and pure hydrogen component; the synthetic spectrum from this model (middle curve) can then be subtracted from the composite spectrum, leaving the bottom tracing - the resultant spectrum of the dwarf carbon component of the binary, which dominates longward of $5000 \AA$. The Swan bandheads of $\mathrm{C}_{2}$ degrading to shorter wavelengths are clearly visible near $5100 \AA$ and $5600 \AA$.

The atmospheric modelling of G77-61 by Gass et al. (1988) is the only attempt thus far to analyze a carbon dwarf; this study must be regarded as exploratory. The formation of carbon-based molecules is greatly enhanced at these high pressures compared with the atmospheres of AGB carbon stars, so the opacity set in this study, must be inadequate. The amount of opacity in AGB carbon stars increases with decreasing wavelength, and there is a strong indication that the same is true for dwarfs. While the fits to the red part of the spectrum in the Gass et al. analysis are adequate, far too much flux is predicted at shorter wavelengths, which must be a direct consequence of the missing opacity. It is obvious that much work remains to be done in order to produce accurate models of this rare but interesting new type of dwarf star.

Acknowledgments. It is a pleasure to thank F. Allard, R. Wehrse and T. Tsuji for illuminating discussions, and F. Allard for some results in advance of publication. The author acknowledges financial support from the National Science Foundation through grant AST 92-17961.

\section{References}

Allard F., 1990, Ph.D. thesis, Univ. Heidelberg

Allard F., 1994, In: P.Thejll \& U.G.Jørgensen (eds.), Poster session proceedings of IAU Coll. 146, Copenhagen Univ., p. 1

Becklin E.E., Zuckerman B., 1988, Nature, 336, 656

Burrows A., Liebert J., 1993, Rev. Mod. Phys., 65, 301

Dahn C.C., Liebert J., Kron R.G., Spinrad H., Hintzen P.M., 1977, Astrophys. J., 216, 757 
Dearborn D.S.P., Liebert J., Aaronson M., Dahn C.C., Harrington R.S., Mould J.,Greenstein J.L., 1986, Astrophys. J., 300, 314

Gass H., Liebert J., Wehrse R., 1988, Astron. A strophys., 189, 194

Green P., Margon B., MacConnell J., 1992, Astrophys. J. Let., 380, L31

Kirkpatrick J.D., Kelly D.M., Rieke G.H., Liebert J., Allard F., Wehrse R., 1993, Astrophys. J., 402, 643

Kirkpatrick J.D., Henry T.J., Liebert J., 1993, A strophys. J., 406, 701

Jones H.R.A., Longmore A.J., Miller S., Tennyson J., 1993, A strophys. J., in preparation

Liebert J., Schmidt G.D., Lesser M., Stepanian J.A., Lipovetsky V.A., Chaffee F.H., Foltz C.B., Bergeron P., 1994, Astophys. J., in press

Lunine J.I., Hubbard W.B., Marley M.S., 1986, Astrophys. J., 310, 238

Markarian B.E., Stepanian J.A., 1983, Astrophyzica, 20, 21

Ruan P., 1991, PhD Dissertation, Australian National University

Warren S.J., Irwin M.J., Evans D.W., Liebert J., Osmer P.S., Hewett P.C., 1993, Mon. Not. R. Astr. Soc., 261, 185

Zinnecker H., McCaughrean M.J., Wilking B.A., 1993, In: Protostars and Planets III, eds. E. Levy, J.I. Lunine, Univ. of Arizona Press, Tucson, in press

Zuckerman B., 1992, In: Planets Around Pulsars, ASP Conf. Series vol. 36, eds. J.A. Phillips, S.E. Thornett, S. Kulkarni, ASP, Provo UT, p. 303

Zuckerman B., Becklin E.E., 1987, Nature, 330, 138 